\title{
The Effect of Self-Diffusion on the Zn Diffusion in III-V Compound Semiconductors
}

\author{
Liangliang Tang ${ }^{1,}$, Chang $\mathrm{Xu}^{1}$, Zhuming Liu ${ }^{2}$ \\ ${ }^{1}$ College of Energy and Electricity, Hohai University, Nanjing, China \\ ${ }^{2}$ Institute of Electrical Engineering, Chinese Academy of Sciences, Beijing, China
}

Email address:

tangll@hhu.edu.cn (Liangliang Tang)

${ }^{*}$ Corresponding author

\section{To cite this article:}

Liangliang Tang, Chang Xu, Zhuming Liu. The Effect of Self-Diffusion on the Zn Diffusion in III-V Compound Semiconductors. International Journal of Materials Science and Applications. Vol. 5, No. 5, 2016, pp. 228-234. doi: 10.11648/j.ijmsa.20160505.18

Received: September 21, 2016; Accepted: October 10, 2016; Published: October 13, 2016

\begin{abstract}
Zn diffusion in III-V compound semiconductors are commonly processed under group V-atoms rich conditions because the vapor pressure of group V-atoms is relatively high. In this paper, we found that group V-atoms in the diffusion sources would not change the shaped of Zn profiles, while the Zn diffusion would change dramatically under group III-atoms rich conditions. The $\mathrm{Zn}$ diffusions were investigated in typical III-V semiconductors: GaAs, GaSb and InAs. We found that under group V-atoms rich or pure $\mathrm{Zn}$ conditions, the double-hump Zn profiles would be formed in all materials except In As. While under group III-atoms rich conditions, single-hump $\mathrm{Zn}$ profiles would be formed in all materials. Detailed diffusion models were established to explain the $\mathrm{Zn}$ diffusion process; the surface self-diffusion of matrix atoms is the origin of the abnormal $\mathrm{Zn}$ diffusion phenomenon.
\end{abstract}

Keywords: Zn Diffusion, Self-Diffusion, III-V Compound Semiconductors

\section{Introduction}

$\mathrm{Zn}$ diffusion into III-V compound semiconductor is an important process for fabrication of junction field-effect transistors [1], thermophotovoltaic (TPV) cells [2-5] and infrared detectors [6]. The shape of $\mathrm{Zn}$ profiles has a direct impact on device performance. However, under constant $\mathrm{Zn}$ pressure, Zn profiles in III-V semiconductors don't follow the complementary error function [7], distorted phenomenon occurred in $\mathrm{Zn}$ profiles [1-2]; it is difficult to predict the precise diffusion profiles. During $\mathrm{Zn}$ diffusion process, the self-diffusion of matrix atoms occurred at the same time, thus the self-diffusion should be taken into account during $\mathrm{Zn}$ diffusion.

Take typical III-V semiconductor - GaAs for example, the vapor pressure of As is higher than the Ga, thus the As atoms are commonly added into the diffusion source. Under As-rich conditions, the double-hump $\mathrm{Zn}$ profile is obtained in GaAs [1]. In this paper, we found that if Ga atoms were added into the diffusion source, the first hump in $\mathrm{Zn}$ profile was "removed". Based on the above phenomenon, the investigations were extended to the materials of $\mathrm{GaSb}$ and InAs. The surface self-diffusion of matrix atoms should be responsible for the abnormal $\mathrm{Zn}$ diffusion phenomenon, details about our experiments and analyses are presented below.

\section{Experimental Procedure}

The n-type $\mathrm{GaSb}$ and InAs wafers were selected for the experiments. There are several internal defects such as $\mathrm{Si}_{\mathrm{As}}$ acceptors, $\mathrm{Si}$ clusters, and $\mathrm{Si}_{\mathrm{Ga}}-\mathrm{Ga}$ vacancy complexes in $\mathrm{n}$-GaAs which would have effect on $\mathrm{Zn}$ diffusion [10], thus the semi-insulating GaAs with little defects was selected. The specific parameters of wafers and the diffusion sources are listed in Table 1; the purity of all the diffusion sources is 99.9999\%. 
Table 1. Parameters of wafers and diffusion sources used for experiments.

\begin{tabular}{|c|c|c|c|c|}
\hline \multirow{2}{*}{ Wafer } & \multirow{2}{*}{ Doping/ orientation } & \multicolumn{3}{|c|}{ Diffusion sources } \\
\hline & & Pure Zn & III-atoms rich & V-atoms rich \\
\hline GaAs & $\begin{array}{l}\text { Semi-insulating } \\
<100>\text {-oriented }\end{array}$ & $\mathrm{Zn}$ & $\begin{array}{l}\mathrm{Zn}-\mathrm{Ga} \\
(3 \mathrm{wt} \% \mathrm{Zn})\end{array}$ & $\begin{array}{l}\mathrm{Zn-As} \\
\left(\mathrm{Zn}_{3} \mathrm{Sb}_{2}\right)\end{array}$ \\
\hline $\mathrm{GaSb}$ & $\begin{array}{l}\text { Te-doped (n-type) } \\
<100>\text {-oriented }\end{array}$ & $\mathrm{Zn}$ & $\mathrm{Zn}-\mathrm{Ga}(3 \mathrm{wt} \% \mathrm{Zn})$ & $\begin{array}{l}\mathrm{Zn}-\mathrm{Sb} \\
\left(\mathrm{Zn}_{3} \mathrm{Sb}_{2}\right)\end{array}$ \\
\hline InAs & $\begin{array}{l}\text { S-doped (n-type) } \\
<100>\text {-oriented }\end{array}$ & $\mathrm{Zn}$ & $\begin{array}{l}Z n-I n \\
(3 w t \% Z n)\end{array}$ & $\mathrm{n} / \mathrm{a}$ \\
\hline
\end{tabular}

$\mathrm{Zn}$ diffusion experiments were performed in vacuum sealed ampoules. The sealed ampoules were placed in a small-sized OTF-1200X tube furnace with the temperature control accuracy of $\pm 2 \mathrm{~K}$. The rate of temperature increase was set at $10 \mathrm{~K} / \mathrm{min}$. After the diffusion process, the ampoules were quickly pulled out from the tube furnace and cooled in $\mathrm{N}_{2}$. $\mathrm{Zn}$ profiles in all semiconductors were measured using CAMECA IMS4F SIMS (secondary-ion-mass spectrometry).

\section{Experimental Results and Analysis}

\subsection{Zn Diffusion in GaAs}

Figure 1a shows the $\mathrm{Zn}$ profiles using $\mathrm{Zn}, \mathrm{Zn}-\mathrm{Ga}$ and $\mathrm{Zn}-\mathrm{As}$ alloy sources. Clear disparities can be found. Under pure $\mathrm{Zn}$ condition, the $\mathrm{Zn}$ profile has double humps; while under Ga-rich condition, the $\mathrm{Zn}$ profile has only a single hump, the first hump has been "removed". We found that $D \propto C(D: Z n$ diffusion coefficient, $C$ : $\mathrm{Zn}$ diffusion coefficient) is fit for the main region of the single-hump profile and the second hump of double-hump profile; while $D \propto C^{2}$ is fit for the first hump of the double-hump profile.

The $\mathrm{Zn}$-As alloy is commonly used for the diffusion source due to the high pressure of As [11], while our experimental results showed that the As-rich condition was similar to that of pure $\mathrm{Zn}$. The As atoms in the diffusion sources can only shorten the first hump of the double-hump profile.

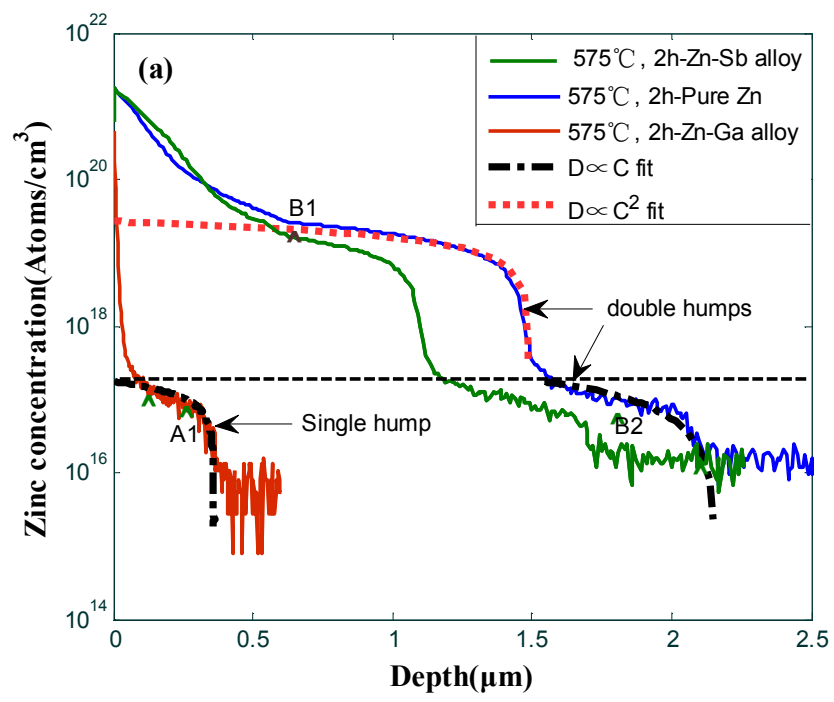

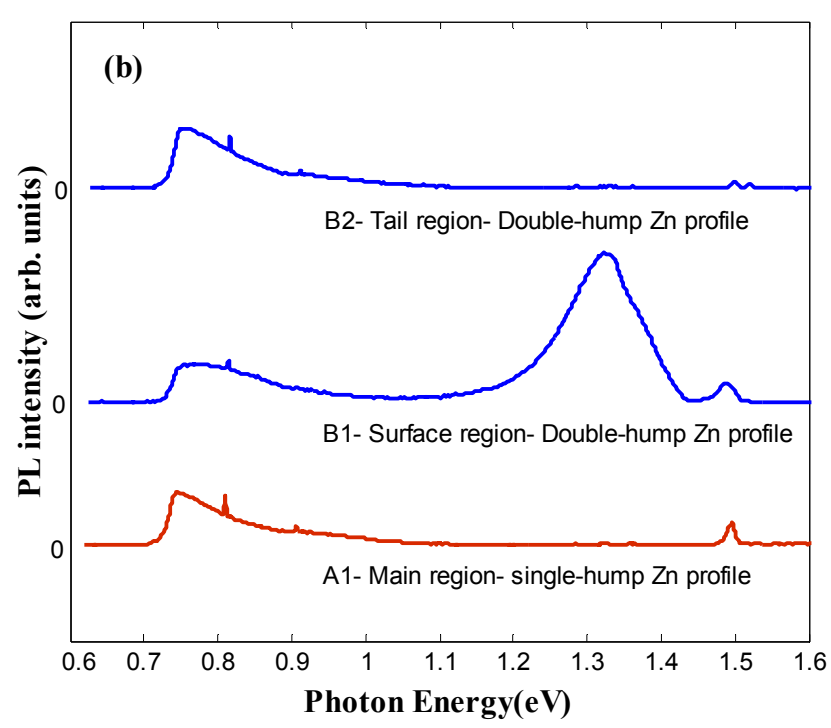

Figure 1. (a) Zn profiles in GaAs with different sources and simulations. (b) $P L$ spectra at $15 \mathrm{~K}$ at the point of $A 1, B 1$ and $B 2$.

Figure $1 \mathrm{~b}$ shows the PL peaks at different etching depth. The main region of single-hump profile (A1) showed the similar PL peaks with the second hump of double-hump profile (B2). The PL peaks at $0.75 \mathrm{eV}$ demonstrate interstitial $\mathrm{Zn}$ toms; the PL peaks at $1.5 \mathrm{eV}$ denote in-band transitions [12, 13]. Compared to the above two regions, another PL peak at $1.32 \mathrm{eV}$ (B1) exists in the first hump of double-hump profile, which represents the $\mathrm{Zn}-\mathrm{V}_{\mathrm{As}}$ complex.

Considering the diffusion and PL results, we may conclude that $\mathrm{Zn}$ diffusion mechanism is determined by diffusion sources. Under Ga-rich conditions, Ga vacancies in the surface of wafers were occupied by Ga atoms. Therefore, $\mathrm{Zn}$ atoms diffuse by kicking out Ga atoms, as shown in Figure 2.

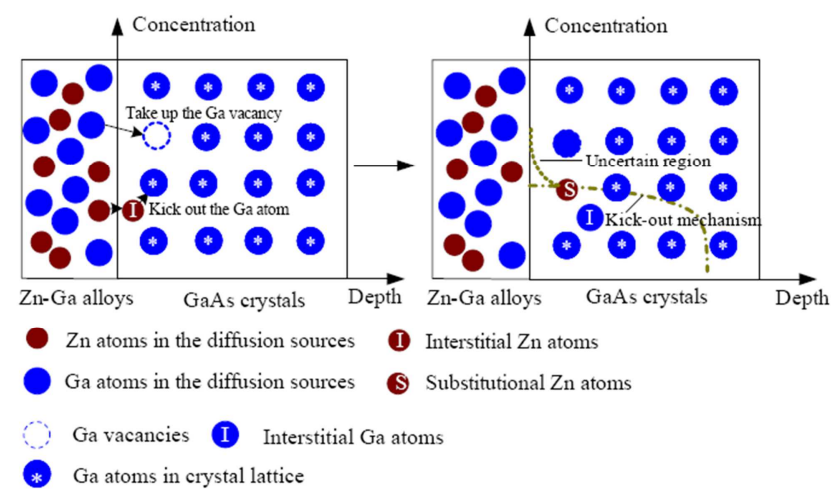

Figure 2. Schematic diagram of the Zn diffusion under Ga-rich conditions. 
The above diffusion can be described as an interstitial $\mathrm{Zn}$ atom $\left(Z n_{i}^{+}\right)$kicking out a $\mathrm{Ga}$ atom $\left(G a_{G a}^{0}\right)$ to the interstitial state $\left(I_{G a}^{+}\right)$:

$$
Z n_{i}^{+}+G a_{G a}^{0} \rightarrow Z n_{G a}^{-}\left(Z n_{s}^{-}\right)+I_{G a}^{+}+h^{+}
$$

The function of $D \propto C$ can be got from Equation 1. The simulated $D \propto C$ profile coincides well with the main regions of the single-hump $\mathrm{Zn}$ profile, as shown in Figure 1a.

Under pure $\mathrm{Zn}$ conditions, Ga vacancies are generated owing to the self-diffusion from the GaAs wafers. Zn atoms would diffuse by taking up the vacancies firstly; therefore the dissociative diffusion would operate in the first hump. This

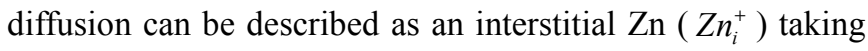
up a Ga vacancy $\left(V_{G a}^{0}\right)$ and turning to an acceptor $\left(Z n_{G a}^{-}\right)$:

$$
Z n_{i}^{+}+V_{G a}^{0} \rightarrow Z n_{G a}^{-}\left(Z n_{s}^{-}\right)+2 h^{+}
$$

The function of $D \propto C^{2}$ can be got from Equation 2, which coincides with the first hump of the double-hump profiles.

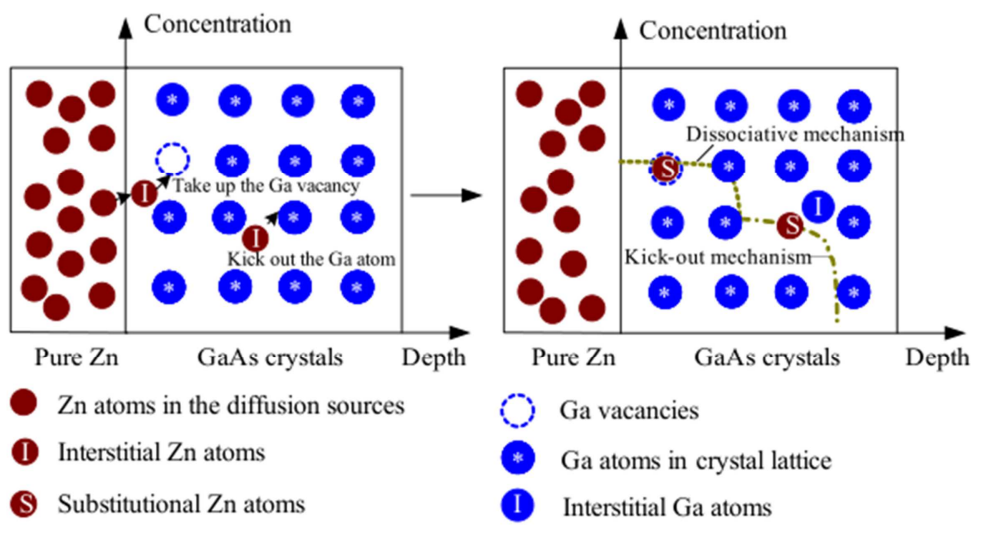

Figure 3. Schematic diagram of the Zn diffusion under pure Zn conditions.

The Ga vacancies were generated by self-diffusion; its concentration would decrease to an intrinsic equilibrium value at the frontier of diffusion profile. The short of Ga vacancies would prevent further $\mathrm{Zn}$ diffusion. Therefore, the $\mathrm{Zn}$ atoms would be compelled to kick the Ga atoms. The overall diffusion process under pure Zn condition is shown in Figure 3.

The diffusion coefficients of double-hump $\mathrm{Zn}$ profiles over the temperature range of $525 \sim 575^{\circ} \mathrm{C}$ are listed in Figure 4 . It is that the relationship of $D \propto C$ fits for all the tail regions of the double-hump profiles, and the relationship of $D \propto C^{2}$ fits for the surface region of the double-hump $\mathrm{Zn}$ profiles. Arrhenius plot of the surface $\mathrm{Zn}$ diffusion coefficients fitted for the tail and surface regions show good linearity, i.e., the distorted phenomenon in double-hump $\mathrm{Zn}$ profiles are discovered. This above diffusion model makes a reasonable explanation double-hump profiles and can be used to make accurate predictions under other diffusion conditions.

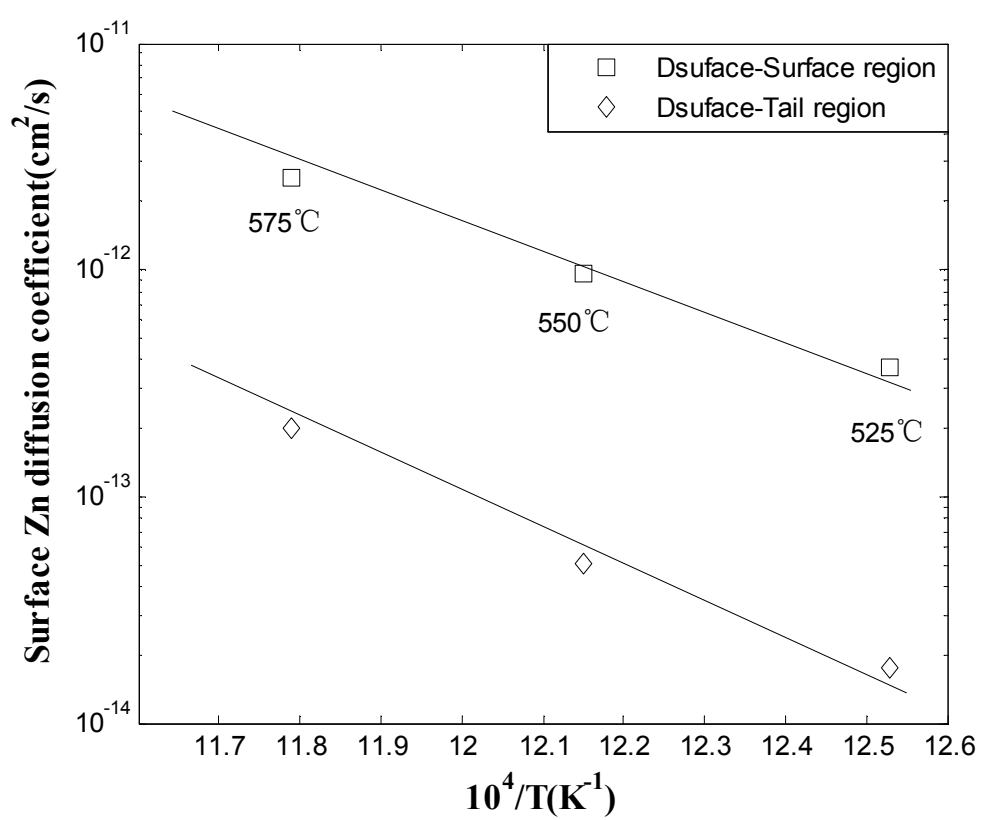

Figure 4. Arrhenius plot of the surface Zn diffusion coefficients fitted for double-hump Zn profiles. 
In summary, the shape of $\mathrm{Zn}$ profile in GaAs is depended by the diffusion source. When $\mathrm{Zn}-\mathrm{Ga}$ alloy sources are used, the self-diffusion of $\mathrm{Ga}$ atoms is hold back, the kick-out mechanism operates owing to short of Ga vacancies, a single-hump profile will be generated. When pure $\mathrm{Zn}$ or $\mathrm{Zn}$-As alloy sources are used, Ga vacancies will generate owing to the self-diffusion. Therefore, the dissociative mechanism operates until the concentration of the Ga vacancies reduced to an intrinsic equilibrium value, after which the kick-out mechanism operated. The different relation between $D$ and $C$ for the above two mechanisms causes the distortion of the $\mathrm{Zn}$ profile, thus the double-hump profile will be formed.

\subsection{Zn Diffusion in GaSb}

$\mathrm{Zn}$ diffusion in $\mathrm{GaSb}$ has been investigated intensively due to the important application in GaSb TPV cells [14-18]. In our previous study, the similar $\mathrm{Zn}$ diffusion phenomenon in $\mathrm{GaSb}$ have been found as that in GaAs [19]. Recently, several auxiliary experiments have been done to prove the impact of the Ga-self diffusion from matrix atoms. In the following, we will show three groups of diffusion results processed at 500 , 550 and $610^{\circ} \mathrm{C}$.

Figure 5a shows the $\mathrm{Zn}$ profiles after diffusion at $500^{\circ} \mathrm{C}$ for $2 \mathrm{~h}$ using different sources. Similar to the $\mathrm{Zn}$ diffusion in GaAs, the $\mathrm{Zn}$-Sb source can only shorten the first hump. While the Zn-Ga alloy sources can "remove" the first hump dramatically; this result will play a direct role for fabricating of GaSb cells. The first hump should be precisely etched to increase the quantum efficiency of GaSb cells $[3,15]$, this etching process could be omitted based on the above results. Our PL analyses (Figure 5b) showed that a PL peak located at $0.78 \mathrm{eV}$, which demonstrated the Ga excess existed in the single-hump profile and the second hump of double-hump profile, while this PL peaks didn't exist in the first hump of the double-hump profile. This demonstrates that the $\mathrm{Ga}$ atoms have been escaped by self-diffusion under pure $\mathrm{Zn}$ or Sb-rich conditions.

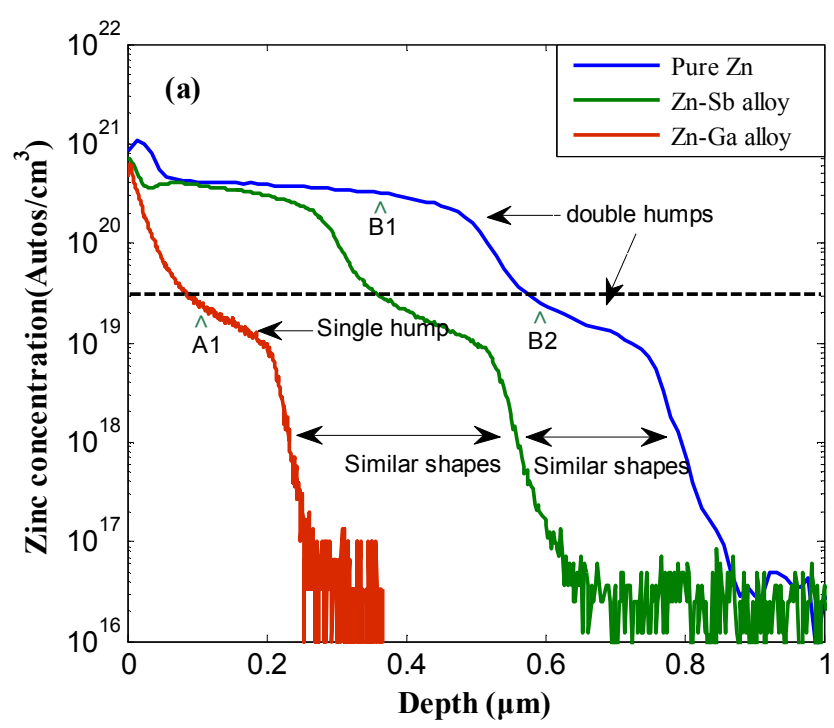

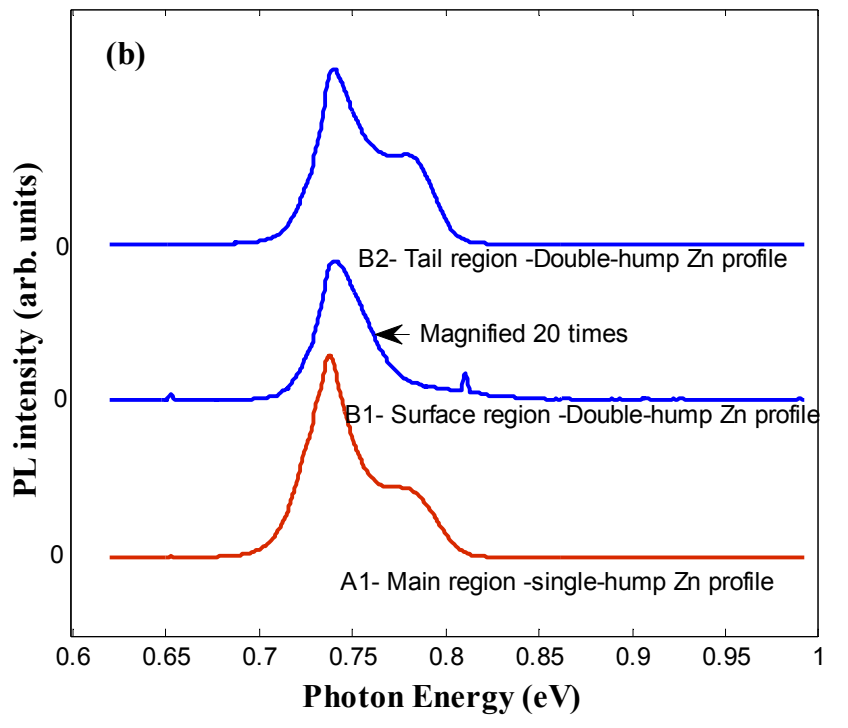

Figure 5. (a) Zn profiles in $n$-GaSb under different sources at $500{ }^{\circ} \mathrm{C}$ for $2 \mathrm{~h}$. (b) $P L$ spectra at $15 K$ at the point of $A 1, B 1$ and $B 2$.

Figure $6 \mathrm{a}$ shows the diffusion results at $550^{\circ} \mathrm{C}$. The $\mathrm{GaSb}$ wafer shows a mirrored surface under Ga-rich conditions, and a single-hump profile is obtained. The GaSb wafer shows the blue surface under the pure $\mathrm{Zn}$ or Sb-rich conditions. Figure 7 shows the surface morphology and elemental analysis for the blue surface; there weren't other elements on the surface except the $\mathrm{Ga}$ and $\mathrm{Sb}$ atoms. This may be a "color center" due to component deviation. This "blue-color center" is similar to a "yellow-color center" in $\mathrm{ZnO}$ when it is heated in vacuum, which causes the deviation of $\mathrm{Zn}$ and $\mathrm{O}$ atoms.

Figure $6 \mathrm{~b}$ shows the diffusion results at $610^{\circ} \mathrm{C}$. The wafer after Ga-rich diffusion conditions also shows a mirrored surface and has a single-hump profile inside. While under pure $\mathrm{Zn}$ or Sb-rich conditions, the wafers are dissolved due to the escaping of large amounts of Ga atoms.

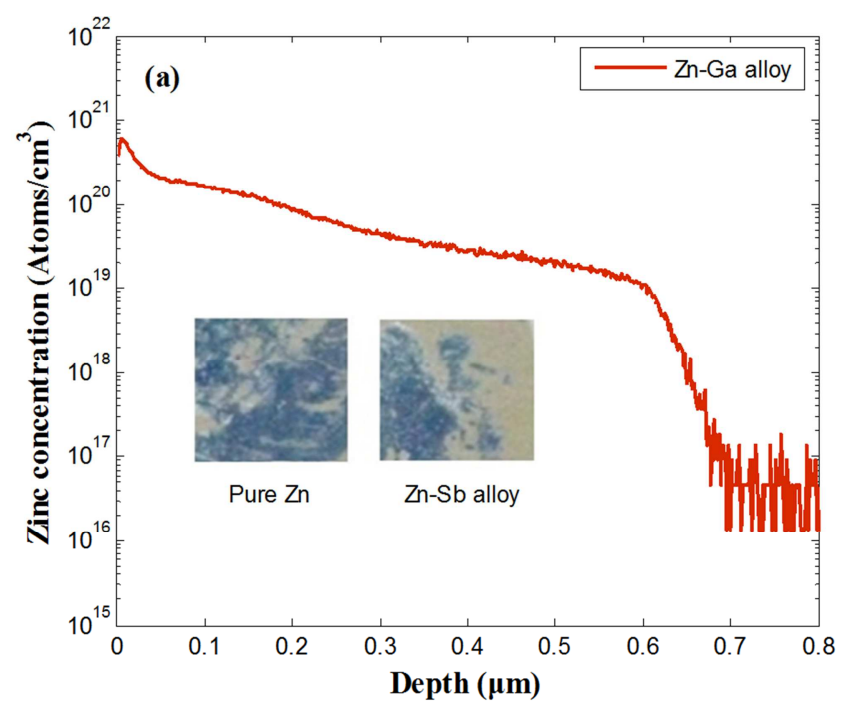




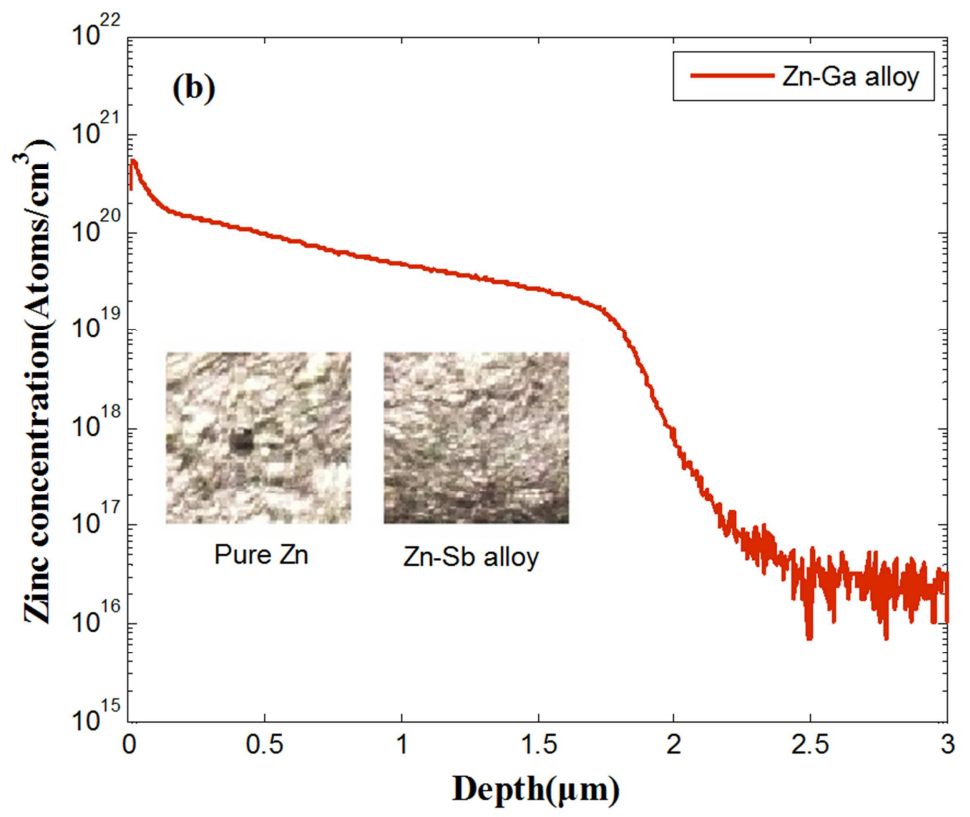

Figure 6. Zn diffusion results in $n$-GaSb under different sources. (a) $T=550^{\circ} \mathrm{C}, 2 \mathrm{~h}$; (b) $T=610^{\circ} \mathrm{C}, 2 \mathrm{~h}$.

(a)
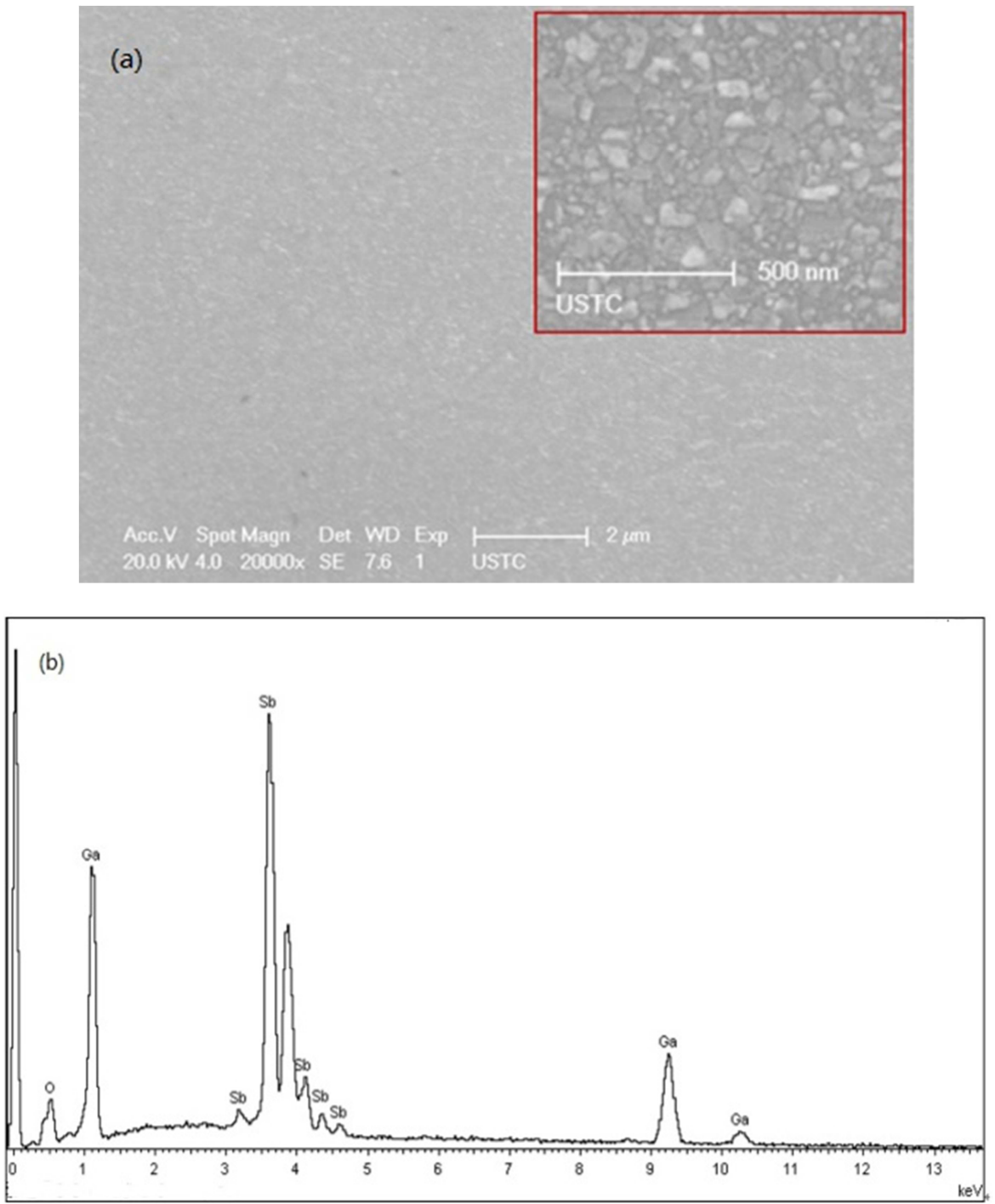

Figure 7. (a) Surface morphology of the GaSb wafer with the blue surface. (b) Elemental analysis. 


\subsection{Zn Diffusion in InAs}

The bandgap of InAs is about $0.35 \mathrm{eV}$, the InAs TPV cells fabricated by the $\mathrm{Zn}$ diffusion method absorb the photons in the range of $1 \sim 3.5 \mu \mathrm{m}[5,20-23]$, thus they have import applications in low temperature TPV systems.

Figure 8a shows the $\mathrm{Zn}$ profiles in $\mathrm{n}$-InAs under Ga-rich conditions. Over $450 \sim 500^{\circ} \mathrm{C}$, all InAs wafers show mirrored surfaces and have single-hump profiles inside. The simulated $D \propto C$ profiles fit well for the profiles and the extracted $\mathrm{Zn}$ surface diffusion coefficient shows the linearity with the reciprocal of temperature (Figure 8c).

Under pure $\mathrm{Zn}$ conditions, the InAs wafer shows the yellow surface above the temperature of $450^{\circ} \mathrm{C}$, which demonstrates that the component deviation occurs and produces the "color center". The temperature is decreased to $440^{\circ} \mathrm{C}$ for comparison, as shown in Figure 8b. Single-hump Zn profiles are obtained under both conditions; this demonstrates that the In atoms can't escape from InAs substrates easily.
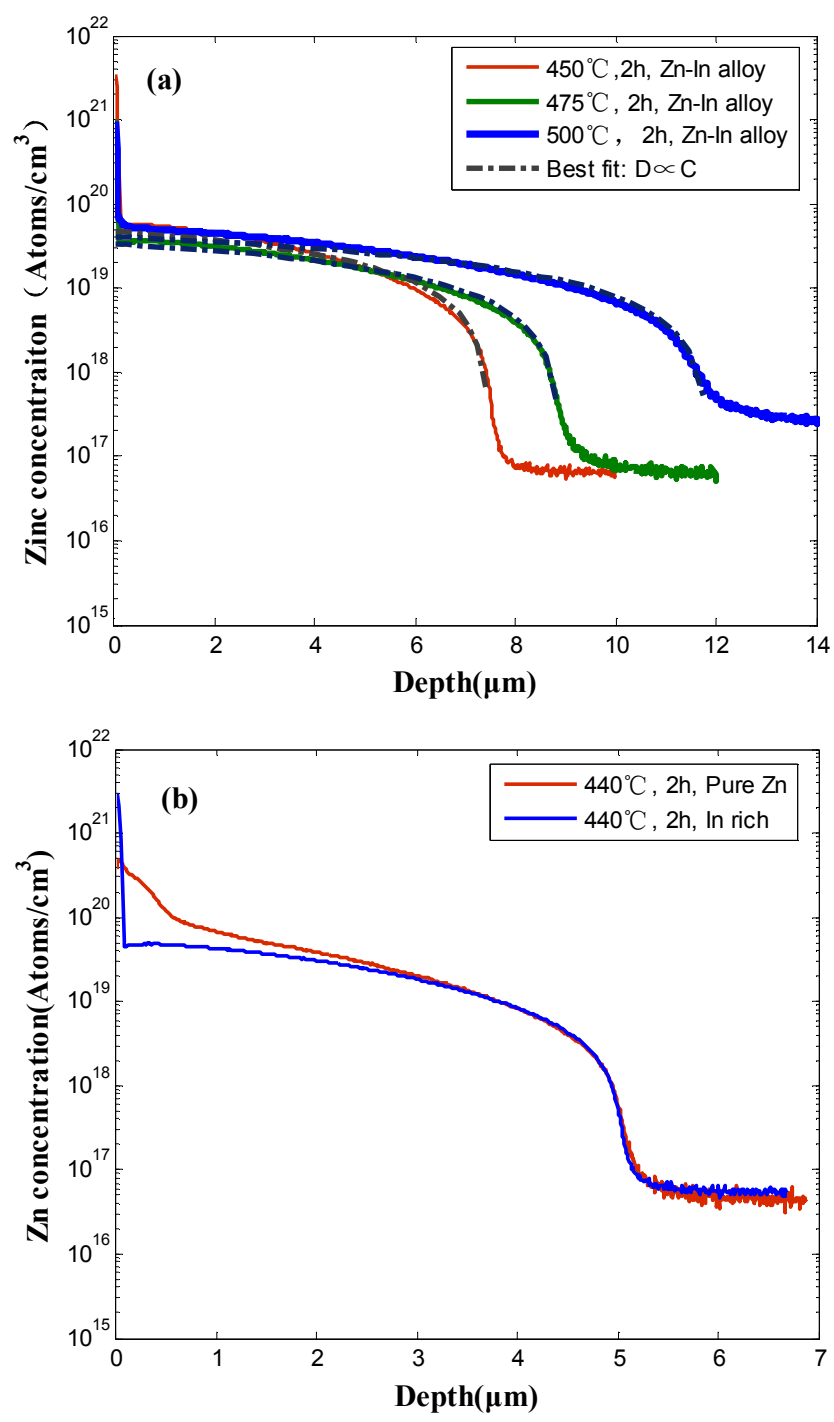

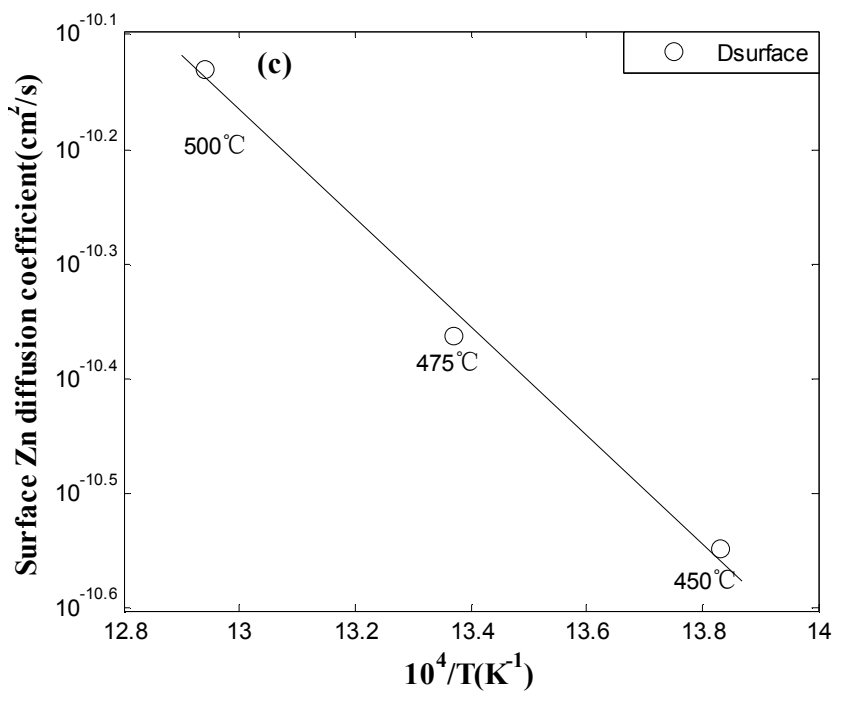

Figure 8. Zn profiles in InAs under (a)Ga-rich and (b) pure Zn conditions.(c) Arrhenius plot of surface $\mathrm{Zn}$ diffusion coefficient fitted for simulating the $\mathrm{Zn}$ profiles under Ga-rich conditions.

\section{Conclusion}

Different diffusion sources are used to study the diffusion mechanisms of $\mathrm{Zn}$ diffusion in III-V compound semiconductors. The III-atoms are crucial to obtain the single-hump $\mathrm{Zn}$ profiles; the diffusion results under V-atoms rich conditions are similar to that of pure $\mathrm{Zn}$ conditions. Under pure $\mathrm{Zn}$ or V-atoms rich conditions, the double-hump profiles will be obtained in GaAs and GaSb. The existing of group III-atoms in diffusion sources would "cut off" the first hump, thus single-hump $\mathrm{Zn}$ profiles would be obtained. The In atoms are stable in InAs substrates, thus the single-hump $\mathrm{Zn}$ profiles would be obtained under pure $\mathrm{Zn}$ or In rich conditions.

\section{Acknowledgements}

This work was funded by the Fundamental Research Funds for the Central Universities (No. 2014B12914), the National Natural Science Foundation of China (No. 51506045) and the Natural Science Foundation of Jiangsu Province, China (No. BK20150805).

\section{References}

[1] S. Reynolds, D. W. Vook, and J. F. Gibbons, "Open-tube Zn diffusion in GaAs using diethylzinc and trimethylarsenic: Experiment and model," J. Appl. Phys., vol. 63, pp. 1052-1059, 1988.

[2] O. V. Sulima, A. W. Bett, M. G. Mauk, B. Y. Ber, and P. S. Dutta, "Diffusion of $\mathrm{Zn}$ in TPV materials: GaSb, InGaSb, InGaAsSb and InAsSbP. In: Fifth Conference of Thermophotovoltaic Generation of Electricity,"pp. 402-413, 2003.

[3] O. V. Sulima, and A. W. Bett, "Fabrication and simulation of GaSb thermophotovoltaic cells," Sol. Energy Mat. Sol. Cells, vol. 66 , pp. 533-540, 2001. 
[4] V. Khvostikov, S. Sorokina, O. Khvostikova, N. K. Timoshina, N. Potapovich, B. Y. Ber, D. Y. Kazantsev, and V. Andreev, "High-efficiency GaSb photocells," Semiconductors, vol. 47, pp. 307-313, 2013.

[5] V. Khvostikov, O. Khostikov, E. Oliva, V. Rumyantsev, M. Shvarts, T. Tabarov, and V. Andreev, "Zinc-diffused InAsSbP/InAs and Ge TPV cells.", Photovoltaic Specialists Conference pp. 943-946, 2002.

[6] E. V. Kunitsyna, I. Andreev, V. Sherstnev, T. L'vova, M. Mikhailova, Y. P. Yakovlev, G. Kaynak, and O. Gurler, "Narrow gap III-V materials for infrared photodiodes and thermophotovoltaic cells," Opt. Mater., vol. 32, pp. 1573-1577, 2010 .

[7] B. Tuck, “Atomic Diffusion in III-V Semiconductors", Hilger, Bristol, pp78-80, 1988.

[8] K. B. Kahen, "Model for the diffusion of zinc in gallium arsenide," Appl. Phys. Lett., vol. 55, pp. 2117-2119, 1989.

[9] K. B. Kahen, J. P. Spence and G. Rajeswaran, "Mechanism for zinc diffusion in n - type gallium arsenide," J. Appl. Phys., vol. 70, pp. 2464-2466,1991.

[10] C. Domke, P. Ebert, M. Heinrich, and K. Urban, "Microscopic identification of the compensation mechanisms in Si-doped GaAs," Phy. Rev. B, vol. 54, pp. 10288-10291, 1996.

[11] N. H. Ky, L. Pavesi, D. Araujo, J. Ganiere, and F. Reinhart, “A model for the $\mathrm{Zn}$ diffusion in GaAs by a photoluminescence study,” J. Appl. Phys., vol. 69, pp. 7585-7593, 1991.

[12] M. Hudait, P. Modak, K. Rao, and S. Krupanidhi, "Low temperature photoluminescence properties of $\mathrm{Zn}$-doped GaAs," Mat. Sci. Eng. B, vol. 57, no. 1, pp. 62-70, 1998.

[13] M. I. Nathan, G. Burns, S. E. Blum, and J. C. Marinace, "Electroluminescence and Photoluminescence of GaAs at $77^{\circ}$ K,” Phy. Rev., vol. 132, pp. 1482, 1963.
[14] V. S. Sundaram, and P. E. Gruenbaum, "Zinc diffusion in GaSb," J. Appl. Phys., vol. 73, pp. 3787-3789, 1993.

[15] G. Rajagopalan, N. S. Reddy, H. Ehsani, I. B. Bhat, P. S. Dutta, R. J. Gutmann, G. Nichols, and O. Sulima, "A simple single-step diffusion and emitter etching process for high-efficiency GaSb thermophotovoltaic devices," J. Electron. Mater., vol. 32, no. 11, pp. 1317-1321, 2003.

[16] A. W. Bett, S. Keser, and O. V. Sulima, "Study of Zn diffusion into GaSb from the vapour and liquid phase," J. Cryst. Growth, vol. 181, no. 1-2, pp. 9-16, 1997.

[17] L. Tang, H. Ye and J. Xu, "A novel zinc diffusion process for the fabrication of high-performance GaSb thermophotovoltaic cells," Sol. Energy Mat. Sol. Cells, vol. 122, pp. 94-98, 2014.

[18] L. Tang, L. M. Farrs, Z. Liu, C. Xu and X. Chen, "Performance improvement of the GaSb thermophotovoltaic cells with n-type emitters," IEEE. Electr. Device, vol. 62, no. 9, 2015.

[19] H. Ye, L. Tang, and K. Li, "The intrinsic relationship between the kink-and-tail and box-shaped zinc diffusion profiles in n-GaSb,” Semicond. Sci. Tech, vol. 28, pp. 015001, 2013.

[20] V. Andreev, V. Khvostikov, O. Khvostikova, N. Kaluzhniy, E. Oliva, V. Rumyantsev, S. Titkov, and M. Shvarts, "Low-bandgap PV and thermophotovoltaic cells." Proceedings of 3rd World Conference on IEEE, pp. 15-18, 2003.

[21] A. Krier, M. Yin, A. R. J. Marshall, et al., "Low Bandgap InAs-Based Thermophotovoltaic Cells for Heat-Electricity Conversion,” J. Electr. Mat., vol. 45(6), pp. 2826-2830, 2016.

[22] A. Krier, M. Yin, A. R. J. Marshall, et al., "Low bandgap mid-infrared thermophotovoltaic arrays based on InAs," Infrared Phys. Techn., vol. 73, pp. 126-129, 2015.

[23] H. Y. Deng, Q. W. Wang, J. C. T, et al., "Electrical property of infrared-sensitive InAs solar cell," Chin. Phys. Lett., vol. 27, no. 11, 116206 (pp. 1-5), 2010. 